\title{
Temores Iniciales de los Estudiantes de Psicología ante el Inicio de la Práctica de la Psicología Clínica
}

\author{
Initial Fears in Psychology Students at the Beginning of their \\ Professional Practice in Clinical Psychology
}

\author{
Horacio Foladori* \\ Universidad de Chile, Chile \\ (Rec: 10 Octubre 2008 Acep: 1 octubre 2009)
}

\begin{abstract}
Resumen
Esta investigación se realiza con estudiantes de psicología en el momento en que deben iniciar la práctica clínica con pacientes. A través de un dispositivo grupal se instala un grupo de reflexión (con técnica de grupo operativo) en torno a los miedos y ansiedades que se desencadenan ante los primeros encuentros con pacientes. El registro de las sesiones permite analizar un cúmulo de preguntas que hacen y se hacen los estudiantes ante esta nueva experiencia profesional y que remiten a su propia identidad. Se presenta también la evaluación grupal al final del proceso. Como conclusiones se destaca la utilidad de esta herramienta para inscribir de forma sana este conjunto de ansiedades así como la imprescindible necesidad de disponer de espacios como éste $\mathrm{u}$ otros para evitar desencadenar procesos psicopatológicos en los estudiantes practicantes.
\end{abstract}

Palabras claves: Grupo de esclarecimiento, grupo operativo, simbolización

\begin{abstract}
This research is done with students of psychology in the moment in which they have to start their clinical practice with patients. Through a group strategy, a reflective group coordinated with the operative group theory is installed. The coordination centers its attention around the fears and anxieties that the students present when they must meet their first patient. Registration of the sessions allows analyzing the questions that the students have about this process, which refers to their own identity. The autoevaluation of the group at the end of the process is also presented. The usefulness of this tool is indicated as a conclusion, because it permits to register in a healthy way the anxieties that are present in the students. Besides, the need of this kind of device is recognized in order to avoid the unleasement of pathological proceses in the student's practice.
\end{abstract}

Key words: Thinking over group, operartive group, symbolization 


\section{Introducción}

La preocupación por la formación de psiquiatras y psicólogos lleva ya larga data. Cuando se habla de formación se está poniendo el énfasis en ciertas habilidades que no se desarrollan en seminarios, ni a través de la lectura de textos, ni en cursos y programas previamente delineados que ofrecen las carreras universitarias. La formación tiene que ver con el desarrollo de ciertas habilidades que forman parte de la manera de ser, de pensar y de sentir, es decir, de la personalidad total de los profesionales indicados. Hay que partir del reconocimiento de un doble registro de aprendizaje: aquel académico por excelencia y otro informal, transversal, actitudinal y de formas de pensar y de sentir, que es producto de un cierto "desarrollo" personal.

Y si bien esta problemática puede ser atingente para cualquier profesión, es particularmente relevante en el caso de los psiquiatras y psicólogos ya que está en juego su salud mental. Dicho de otro modo, se trata de pensar qué ocurre cuando dos o más personas entran en contacto con la tarea de esclarecer y, en todo caso, "curar" cierta sintomatología denunciada como indeseable por el poseedor de la misma. Qué ocurre cuando se inician las entrevistas alrededor de una demanda de atención.

Desde que los dispositivos de psicoterapia de grupo fueron inventados, una de las insistentes preocupaciones de los especialistas tiene que ver con su aplicación a los procesos de formación de profesionales en el área. En estos países de América Latina, se desarrollaron desde mediados del siglo veinte, una amplia variedad de iniciativas tendientes a abordar de diversas maneras los problemas que presenta la formación (no la información) en el terreno de la salud mental. Por ejemplo, Etchegoyen (1959) reconoce que en la Argentina, el primero en utilizar grupos para apoyar la formación de alumnos en psiquiatría ha sido Enrique Pichón-Rivière (1957). Etchegoyen ha realizado experiencias propias con grupos "didácticos" en el marco de la Universidad de Cuyo, utilizando para esa ocasión el modelo descrito por Grinberg, Langer y Rodrigué (1957), quienes lo habían propuesto para estudiantes de medicina. Sostiene que si el estudiante "logra ver los conflictos que operan dentro de sí y sepa reconocer los matices irracionales de su propia conducta, su actitud frente al enfermo y a la sociedad ayudarán".

Bleger (1961) es invitado por la Facultad de Medicina de la Universidad de República, Uruguay, para supervisar la puesta en marcha del nuevo plan de estudios de medicina que contaba en primer año con un espacio de grupos operativos, donde los estudiantes dialogaban acerca de qué era el estudio de la medicina y su relación con el cuerpo.

En Chile, también son muy tempranos los estudios en la materia. Ganzaraín y colaboradores $(1957,1958,1959)$ realizan minuciosos estudios sobre la efectividad de la psicoterapia de grupo aplicada a estudiantes de psiquiatría.
Además de descubrir que el aprendizaje de la transferencia es mucho mejor en aquellos alumnos que han participado en grupos, observa (1959) "la alta frecuencia de afecciones psiquiátricas en los alumnos de medicina”, lo que obliga a buscar en los grupos psicoterapéuticos no solamente un instrumento útil para apoyar la formación de los estudiantes, sino un medio para abordar además los problemas psicológicos que los alumnos presentan.

Otras experiencias tienen lugar en México, con la introducción de grupos psicoanalíticos terapéutico-didácticos en las maestrías de Psicología Clínica (Langer 1977, Cabeza y Foladori 1978).

El interés por la formación de los estudiantes de psicología en Chile, abrió la posibilidad de instalar desde 1993 grupos operativos como espacios de reflexión en diversas universidades del país (Foladori 2001). En la Facultad de Ciencias Sociales de la U. de Chile fue posible además abrir grupos psicoterapéuticos conducidos con técnica psicoanalítica (Foladori 2000).

En realidad el grupo operativo (psicoanalítico) presenta algunas ventajas adicionales a los grupos manejados con la técnica psicoanalítica en el modelo bioniano; entre ellas se encuentra la de favorecer una menor regresión de los participantes y ajustarse a periodos más breves como puede ser la lógica de los semestres universitarios. Puede tener un objetivo de aprendizaje, un objetivo de esclarecimiento (Ulloa 1973) y uno terapéutico según el tipo de experiencia que se desee implementar. En general, se lo ha utilizado con la finalidad de aprendizaje de los procesos grupales, sin embargo hay antecedentes de que había sido utilizado (Foladori, 1984) para facilitar el entrenamiento en entrevista abierta.

En las experiencias que se realizaron en la U. de Chile y que se da cuenta por medio de esta comunicación, se retoma la idea del grupo de esclarecimiento focalizándose en las diversas ansiedades que presentan los estudiantes cuando se ven en la necesidad de iniciar sus primeras entrevistas clínicas. Se le llama de esclarecimiento (Dellarossa, 1979) porque el dispositivo está dispuesto para pensar acerca de una situación que aparece inicialmente como confusa. En muchos casos, dicha confusión tiene que ver con un conglomerado poco diferenciado de los afectos más dispares, efecto de re-actualizaciones que se producen en los participantes por tener que enfrentarse a situaciones desconocidas. El espacio de reflexión permite identificar tanto las ansiedades que emergen como las temáticas que las producen, introduciendo cuando se logra producir luz en la confusión, una cuota de alivio en los participantes de la experiencia. Al respecto, Sarreta y Veloz (1989) destacan a partir de su propia experiencia con el grupo de reflexión que éste parece haber desempeñado "el importante papel de contención de ansiedades muy intensas que son propias de los grupos y que deben ser tenidas en cuenta mientras se lleva a cabo la formación psicoterapéutica". Sostienen 
que se le ha brindado a los estudiantes un espacio mental diferente del consultorio "donde pudieron expresar y tomar conciencia de fenómenos psíquicos que toman una fuerte vigencia en esos momentos y que pueden obstaculizar el aprendizaje o aun impedirlo totalmente".

Es decir, en la experiencia de estos autores el grupo de reflexión que se desarrolla paralelamente a cursos y seminarios, tiene un destacado papel para posibilitar un aprendizaje más efectivo, profundo, y donde los obstáculos epistemofílicos pueden ser superados al momento en que se presentan.

No se pude decir que el grupo de reflexión sea terapéutico en stricto sensu, ya que ni se presenta como psicoterapéutico ni persigue objetivos terapéuticos. Ello no quiere decir que no pueda tener efectos psicoterapéuticos por cuanto el esclarecimiento paulatinamente logrado repercute en la modificación de actitudes.

\section{Método}

Lo que se mostrará en este trabajo serán algunas interrogantes que están presentes en los estudiantes que están a punto de iniciar su práctica clínica. Se ha preferido respetar las manifestaciones de los propios estudiantes ya que es a partir de dicha materialidad que las inferencias son posibles. En tal sentido se ha tomado como base lo que técnicamente denominamos emergentes grupales, que tienen la propiedad de vincular temáticas a temores y ansiedades y a su vez, a fantasías que deben ser consideradas. Este terreno enormemente espinoso, no debe tomarse como exagerado en momento alguno. Todas son preguntas o temáticas que todo alumno se formula en la situación. El estudio que Marie Langer (1948) realiza para el caso de analistas principiantes es sumamente esclarecedor acerca de temores, ambivalencias y formas distorsionadas de operar, incluso para aquellos que han tenido varios años de psicoanálisis personal.

\section{El modelo grupal}

El inicio de la tarea clínica por estudiantes de los últimos años de pre-grado conlleva la movilización de importantes ansiedades como lo han señalado los diversos autores ya mencionados. Debe destacarse que si bien estas investigaciones se han realizado desde el marco teórico del psicoanálisis, todos los estudiantes que se enfrentan a la situción de entrevista con pacientes, presentan esta gama amplia de preocupaciones, independientemente de la afiliación teórica en la que se inscriben. La subjetividad de los estudiantes es producto de la situación más general de entrevisa clínica (se podría pensar que en cualquier rama de la psicología estaría también presente) y el marco teórico de referencia poco puede hacer para "neutralizar" los sentires que se hacen presente en los estudiantes. Más aún, como los marcos referenciales han sido aprendidos muy recientemente, son vividos más como exigencia que como una verdadera ayuda para abordar la situación clínica. Se abundará sobre este punto más adelante.

La técnica empleada en esta ocasión es la del grupo operativo fundada por Enrique Pichón-Rivière (1971) durante la década del 50. Los grupos se conforman con una docena de estudiantes de cuarto y quinto año que además han elegido la rama clínica de su interés para comenzar a entrevistar pacientes y, eventualmente, iniciar procesos psicoterapéuticos. Los estudiantes cuentan con clases teóricas y con espacios de supervisión para reflexionar acerca del proceso de instalación del proceso psicoterapéutico. Sin embargo, la Universidad no dispone curricularmente de un espacio apropiado para abordar lo que ocurre en el propio mundo interno de los estudiantes, considerando, tal vez, que dicho espacio no es necesario o que se inscribiría en la imprescindible psicoterapia personal que los estudiantes debieran realizar en un espacio extra-universitario. Pareciera que a la institución le cuesta hacerse cargo de este tipo de temáticas y de abordar las preguntas que se deducen de las mismas.

Así, este espacio nuevo es ofrecido como curso electivo por el académico, en el que se inscriben estudiantes que han reconocido que sus primeros pasos en la clínica son productores de ansiedades diversas. El grupo operativo fuciona durante un semestre con sesiones de una hora y media semanal, siendo coordinado por un equipo de especialistas según el modelo clásico (Foladori, 1999, 2001) para el funcionamiento de esta técnica $\left(^{1}\right)$. La consigna que se plantea para el trabajo del grupo focaliza el análisis en el abordaje de la situación clínica que los estudiantes tienen por delante. Así, el grupo está en condiciones de asociar libremente acerca de la tarea clínica, produciéndose un discurso grupal que es interrogado por la coordinación para facilitar su reflexión y producir su esclarecimiento.

\section{Las temáticas y sus ansiedades.}

El recorrido que se realizará tanto por las temáticas como por las ansiedades que las mismas connotan, no supone ordenamiento alguno, ya que cada grupo tiene su "estrategia" particular para abordar los obstáculos en un proceso que nada tiene de lineal. Por esta razón, la necesidad expositiva simplemente indiza las problemáticas. Ello no quiere decir tampoco que el grupo pueda comenzar por abordar cualquiera de ellas, ya que el esclareciento de las preguntas iniciales no sólo funciona como un factor que disminuye la ansiedad ante las siguientes sino que también hay que reconocer que algunas temáticas requieren mejorar

\footnotetext{
El autor no solamente ha implementado estos grupos semestrales, sino que también ha supervisado, durante varios años, grupos realizados por otros colegas, por lo que tanto los ejemplos como las reflexiones provienen del material producido en varias experiencias.
} 
los grados de aceptación de la propia patología (conciencia de enfermedad) para poder ser reconocidas como tales.

Tal vez la asignación al otro de la etiqueta de "paciente" resulta en un mecanismo tranquilizador inicial, ya que el implícito de los estudiantes se podría formular como "No soy yo el que está enfermo sino ese otro que tengo enfrente".

Dicha fórmula, la de tener alguien "enfrente"(el paciente) al que hay que "enfrentar", supone de entrada una oposición estéril, pero que conserva buena parte de la frágil tranquilidad requerida para poder meterse en la situación. Enfrentarse a lo desconocido, al desconocido, constituye una actitud defensiva paranoide que entra a minar la relación de rapport inicial. Más tarde, salvadas múltiples dificultades, los estudiantes podrán reflexionar acerca del "trabajo en común a realizar con el paciente", lo que supone ya la elaboración de la ansiedad del comienzo.

\section{La primera entrevista, "la primera vez".}

La "primera entrevista" es el tema que preocupa y ocupa durante varias sesiones ya que se constituye en una experiencia y como tal es inasible por medio de relatos. La experiencia se tuvo o no se tuvo, no hay allí medias tintas. Pero aquel que la tuvo y que, por tanto, ya pasó por ella - como si fuese una prueba de fuego o rito de iniciación - es poseedor de poder, de un estatus y de un saber que ocasiona no pocas envidias en su entorno. Aquellos que no pasaron por ella aún, tratarán de imaginársela poniendo en juego todo tipo de experiencias anteriores -de similar estructura-, estereotipos y prejuicios acerca de lo que allí pudiera ocurrir.

Se ve desfilar por dicha temática, ítems específicos que llegan a través de preguntas vinculadas a las fases previas (llamadas telefónicas, modalidades para fijar la cita, reserva del espacio de consulta y "adecuación" de éste, distribución de los espacios de "psicoterapeuta" y "paciente" enfrentados o no, dónde se sienta cada quien, etc., etc. Algunos de estos aspectos volverán a replantearse a propósito de otras temáticas. Es visible el deseo de que "la cosa resulte" sin importar mucho al principio lo que allí ocurra, y el "adelanto" a la frustración de "si el paciente falta", casi como deseando que no concurra.

No es difícil descubrir que la "primera entrevista" es vivida con aquellas ansiedades y preguntas autoformuladas cuando "la primera vez", mostrando el intenso contenido sexual que aparece transferido a la entrevista psicológica. $\mathrm{Al}$ ser ello señalado, aparecen innumerables recuerdos alusivos, dudas y sobre todo miedos por el daño posible que puede producirse en la situación de encuentro.

Daño a uno mismo y daño al otro. El temor a dañar al paciente está siempre presente de uno u otro modo. Pero también figura allí la ingenuidad perdida, la identidad perdida (ya no se es estudiante sino que profesional), la seguridad perdida (estar solo ante un otro), la teoría perdida (que no sirvió para garantizar la experiencia), etc.

"Al final, no fue tan tremendo", señala un integrante recordando su iniciación, como para calmarse ante la inminencia de su "primera" entrevista.

\section{El asunto de la edad.}

La corta edad de aquellos que ocuparán el rol de psicoterapeutas (20 a 23 años) constituye un tema de honda preocupación. Habría que partir por señalar que se hace difícil separar el asunto de la edad de aquel del rol. El rol es técnico, por tanto importa allí dilucidar las ansiedaes para su ejercicio, pero la edad alude a "experiencia de vida", vale decir, esa supuesta sabiduría que se le otorga a los ancianos. Se piensa en términos acumulativos, cuantitativos, como si fuesen "horas de vuelo", más que aquello que hace al lugar terapéutico que tiene más que ver con la posibilidad de pensar acerca del discurso del otro - dicho en términos muy generales -. Esta diferencia es la que hay que introducir para que el grupo pueda reflexionar sobre sus alcances y derivaciones. yo?"

"¿Qué voy a hacer si el paciente es mucho mayor que

Porque lo que está presente allí es la idea de entrevistar a los propios padres, es esa inversión de lugares que implica tener que asumir responsabilidades ante las cuales no se sienten en condiciones de poder hacerlo todavía. Convertirse en padres de sus propios padres puede ser una fantasía universal, pero ¡qué complicado se torna cuando se realiza el deseo! Y más aún para estudiantes que son dependientes de sus padres, sostenidos y mantenidos por sus padres y con cierta vergüenza en muchos casos de tener que reconocer su dependencia económica, para no reconocer aquella afectiva.

Y vuelve a aparecer vinculado allí el tema del daño, ya que sus sentimientos más agresivos y reprimidos hacia sus padres podrían hacerse presente en la situación de la entrevista, oficiando el "paciente" el lugar de una descarga vengativa, ansiada y temida a la vez.

La inquietud por la "corta" edad se dibuja en algunos ejes. El que primero irrumpe plantea la cuestión del saludo ya que se siente con acierto que la forma del saludo podría determinar el tipo de relación en la entrevista. Formalizar la relación parte por formalizar el saludo. Se plantea así la necesidad de diferenciar la "relación social" de la "relación psicoterapéutica", las normativas que rigen a una y a la otra.

El problema ahora cobra dimensiones cuando aparece la pregunta "Y ¿si es un joven como uno?" Dicho de otro modo, ¿cómo sostener una distancia, una relación formal, cuando el otro se aparece como uno mismo, cuando tiene los mismos problemas que uno, los mismos sufrimientos? ¿Cómo hacer para no fundirme en el otro, con-fundirme? 
El saludo además tiene que ver con la admisión ("cómo lo recibo") y el final de la entrevista ("cómo lo despido"), mostrándose sobre todo el temor a "no poder terminar" (nueva referencia al coito) la entrevista. Un saludo final que podría contener tanto la dificultad ante la despedida como la despedida agresiva y abrupta, cortante.

El acto formal del saludo encubre los afectos en juego ya que puede resultar imposible controlar el "clima" de la relación: el continuo frío - cálido. En ese punto sienten que no están preparados para dilucidar los intentos de seducción que pueden hacerse presentes y sobre todo "situaciones mas calientes", donde en la fantasía no habría límite posible con el caos de la locura. La presencia de lo erótico está mas cerca de los impulsos; su reflexión instalará las condiciones para que lo erótico no se constituya como defensa ante el compromiso en el espacio de la entrevista.

El segundo eje que se hace visible tiene que ver con el estatuto del cuerpo y su apariencia: la vestimenta. “Cómo hacerse más viejo?" es la interrogante que despliega una preocupación por la apariencia. Qué ponerse, qué tipo de vestido o saco utilizar, cómo pintarse, son fórmulas donde las mujeres dejan ver más claramente sus ansiedades. Los varones se inquietarán por el peinado ("¿Se puede hacer una entrevista peinado como Bob Marley?"). Nuevamente, está allí presente la ansiedad de pérdida de su mundo juvenil dependiente y la desautorización que ellos mismos realizan de sus modas y diseños.

Comienza a cobrar sentido que el ejercicio de la psicología clínica configura en la fantasía para los estudiantes la ruptura con su vida "feliz" y el ingreso - a la fuerza - a un mundo adulto desconocido. El miedo parece cabalgar sin control posándose cada tanto en los puntos de contacto con una realidad difícilmente reconocible.

\section{La cuestión de la apoyatura teórica.}

La situación de la entrevista coloca al estudiante frente a un dilema. Ha estudiado no pocos años, diversas asignaturas que han contenido extensas bibliografías. Es de suponer que todo ese tiempo recorrido e invertido algún sentido tendrá cuando se vea enfrentado a la cruda realidad de la entrevista. Y el hecho es que siente que todo lo estudiado y aprendido sirve para casi nada cuando está frente a un otro angustiado y que clama por ser escuchado y por respuestas. No existe, en su parecer, manera alguna de que lo teóricamente aprendido, tenga alguna utilidad para calmar y encauzar lo que la situación de encuentro introduce de manera cualitativa. Hay allí algo que no figuraba en los libros, hay allí una experiencia que debe ser aprehendida ( si desea continuar realizando entrevistas y trabajo clínico) sin que haya contado con antecedentes que le posibiliten recorrer el nuevo encuentro como "conocido". El asunto es cómo enfrentarse a lo nuevo, con qué material viejo.
Es probable que esta vivencia de los estudiantes cuestione la forma de aprender psicología, los planes de estudio, etc. Pero lo que aquí interesa es esta distancia que la situación genera, incluso con los marcos teóricos más amados, al punto de haberse constituido en dogmas. El alumno podrá estar en condiciones de "recitar" textos clínicos completos, pero su indefensión es total cuando se halla frente a un otro, en un espacio que le resulta claustrofóbico. "Nada de lo que aprendí me sirve para hacer una entrevista", ya que no hay experiencia previa, de clase, similar, que pueda situar como modelo, para pensarse en el lugar de entrevistador. Y de la misma forma que es imposible saber qué es el fuego hasta que la persona no se queme, de igual modo las entrevistas "vistas" y realizadas por profesores nada le aportan a su experiencia singular; por el contrario, eso que vieron no es posible replicarlo en "su" entrevista. Y peor aún, los ejemplos "vistos" operan como esquemas que deben ser seguidos rígidamente y que le impiden, a su vez, sostener una cierta flexibilidad para poder escuchar lo que el otro tiene que comunicarle. No hay de momento identificación posible, todo se transforma en un "como si" bizarro que denuncia la impostura y refuerza en muchos casos un superyo sádico que le grita al oído: "Tú no sirves para esto".

Ha de abandonarse entonces la ilusión de que se sabe, la ilusión de que como está en los años finales de la carrera será prontamente psicólogo, la ilusión de control de sí mismo y por tanto, del devenir de la entrevista, la ilusión de utilidad de un aprendizaje libresco, la ilusión de entender...cómo algo del orden de la vida, se ha perdido en tanta justificación erudita.

Pero a su vez, se abre el fantasma de la fragilidad, de la vulnerabilidad, del no saber qué hacer y quedar expuesto sin protección alguna ante un otro que es siempre vivido como amenazador, de ser visible su no saber, sus preguntas por su supuesta vocación.

\section{El temor a ponerse a llorar. El descontrol emocional.}

Para muchos estudiantes el espacio de la entrevista implica una prueba de fuego: probarse a sí mismo. Es decir, van a dar examen; más aún, van a comprobar si pueden dar examen. El encuentro con un otro en un espacio cerrado los deja a merced del otro, ya que se les ha inculcado de manera sistemática que ese es un espacio para el demandante, no para que el entrevistador relate situaciones personales. Se da por sentado que el estudiante ha estado antes - como demandante - en una situación de entrevista y ello no siempre ha sido así, tal vez en la mayoría de los casos no ha sido así. Por tanto, para los entrevistadores que no ha sido así, hay algo que debe ser reprimido, que debe ser excluido del espacio de la entrevista, según normativas técnicas aprendidas "en clase". 
Por ende, quedar expuesto al discurso del otro, al sufrimiento del otro, no es poca cosa cuando no se tiene ni la más remota idea de qué hacer con eso.

Se hace presente una cierta idea de que el sufrimiento del otro pudiera interrogar al propio, pudiera desencadenar - a pesar de no quererlo - un llanto que no sería capaz de contener y menos evadir. Ponerse a llorar con el paciente haciéndose cargo de sus problemas que pudieran ser mis problemas. En todo caso, está allí la dificultad de recortar los espacios, de mantener cierta distancia, de poder pensar acerca de lo que allí ocurre. Algunos dirían que se actúa la transferencia. Pero ¿cómo va a hacer el estudiante para manejarse en dicho medio cuando recién está aprendiendo?

El problema no se ubica en el plano de la realidad; es decir, en los hechos, en la situación de entrevista, en las primeras entrevistas, la gran mayoría no se pone a llorar. El asunto tiene importancia porque opera antes de la situación: es el miedo a ponerse a llorar, es la fantasía de desborde, de descontrol emocional que podría llegar a producirse y ante la eventualidad, se pone de manifiesto la inoperancia de ciertos mecanismos defensivos, la presencia de sentimientos infantiles y formas de reaccionar primitivas que darían cuenta de lo impropio para realizar entrevistas.

Es extraño pero los estudiantes no se plantean sistemáticamente la presencia de todos los afectos básicos. El énfasis está puesto en la tristeza, no en la rabia, sí algo en el amor erótico. Pero esta situación de seducción no es vivida como trágica sino como cómica, poniendo en juego mecanismos de negación (maníacos) por medio de los cuales desmerecen lo incontrolable de la situación. Tal vez también la desean como una manera de sentirse tomados en cuenta.

El temor al llanto (también deseo de poder dar rienda suelta al propio llanto, envidia del propio paciente, podría decirse) y el miedo a no poder detenerlo una vez iniciado, pone sobre alerta acerca de ciertos núcleos melancólicos en los entrevistadores, que podrían activarse en la situación de entrevista. En todo caso, el llanto llama al continente, a la acogida necesaria para poder descargarse, a que haya una función madre que contenga la tristeza presente que la situación desencadena.

\section{La situación de intermediario. “ ¿Cómo me presento?"}

Recibir a un paciente en el espacio del consultorio, aunque este espacio sea el Centro de Atención Psicológica de la Universidad u otra entidad oficial del Ministerio de Salud o municipal, se presenta al estudiante inicialmente como un problema formal. ¿Quién soy? o ¿qué soy? Porque en la medida en que aún no se ha titulado no puede presentarse como El Psicólogo. Tampoco puede hacerlo como El Estudiante, ya que dicha categoría no tiene estatuto profesional; teme, si su realidad es descubierta, que el paciente no desee "atenderse" con él o que desvalorice su presencia. No han sido pocos los académicos que haciendo una defensa exagerada y rígida de la profesión les han prohibido manifiestamente presentarse como psicólogos, mientras no lo sean.

Este lugar intermedio no tiene soporte. Tampoco puede presentarse como "estudiante en práctica" ya que dicho nombre lo remite a un desplazamiento de la demanda: soy yo como estudiante que requiero hacer práctica para entrenarme, por tanto el interés de que esta entrevista tenga lugar, está en mí más que en Ud. Tampoco sirve de nada explicarle al paciente que su trabajo está siendo supervisado - en el mejor de los casos - ya que dicha información no aporta a la definición de ese lugar intermedio. Por supuesto, este lugar indefinido es fuente de temores y ansiedades ya que inevitablemente lo conduce a la pregunta de quién soy yo.

Cuesta comprender - y allí se ha de centrar el trabajo de esclarecimiento - que la pregunta por el ser no puede ser contestada en abstracto, sino a partir de la pregunta por el hacer. Dicho de otro modo, soy en función de aquello que hago, ya que va a ser el lugar en la relación lo que definirá una determinada función para cada uno de los participantes.

Por tanto, la atribución formal (psicólogo, psicoterapeuta, etc.) es posterior a la asunción de la función necesaria.

Estructuralmente hablando, cualquier individuo que le cuenta a otro sus problemas lo construye en la función de escucha (podríamos decir psicoterapeuta), tenga o no los títulos habilitantes para ello. Por eso, hay una función de psicólogo (como lugar del escuchante) que se corresponde con la función de hablante que asume espontánemante el demandante (paciente) cuando se realiza una entrevista. Dicha función de escuchante se instala más allá de todo parámetro formal (psicólogo titulado); en los hechos, a ningún paciente se le ocurre preguntar - en un marco institucional - si aquel que lo recibe está titulado o no. De igual manera funciona la relación médico-paciente; la sola presencia del delantal "hace médico" a la persona que uno tiene por delante.

Esto hace que la función de escuchante sea cumplida por innumerables personas en la sociedad, la gran mayoría de las cuales no son psicólogos titulados.

El estudiante ha realizado la función de escuchante en innumerables ocasiones de manera espontánea, pero ahora debe realizarla explícitamente como "psicólogo", lo que no deja de causarle problemas porque en su sentir se encuentra usurpando un lugar que no le es propio, no se siente validado en ese lugar. Usurpa un lugar jerárquicamente superior, lugar de poder, lugar del padre. El espacio intermedio no le ofrece garantías de identidad, por lo que teme ser preguntado por su afiliación. Es el problema de cómo hacer para no mentir ante una interrogación que no sabe a su vez cómo responder. No es aún adulto como para poder realizar esa función. 


\section{El temor al "contagio" de la locura}

Esta temática está siempre presente, aunque con diversos grados de conciencia sobre sus alcances. Podría pensarse que está presente incluso desde antes que el estudiante se haya decidido por estudiar psicología. No pocos autores han sostenido que esa es la razón profunda de su decisión vocacional, o como se dice popularmente "hay que estar bastante loco para estudiar psicología o psiquiatría".

Esta idea supone que la exposición a la locura desencadenaría a su vez un estado de locura. La idea inicial es de contagio ya que se parte del principio de que el que entrevista está "sano" y el "paciente" es el enfermo. La esperanza es que se pueda mantener dicha separación de manera permamente; sin embargo, el estudiante intuye que las cosas no son tan simples. No se trata de mantener la asepsia de la situación para garantizar un no contagio. Se trata de algo "inevitable", como si la locura terminara imponiéndose a toda costa, no hay forma de pararla. No hay vacuna.

Al entrar a su análisis es posible dilucidar que no se trata de un contagio sino de un "despertar": lo que el "paciente" desencadena con su sufrimiento es una explosión del propio sufrimiento del entrevistador. Ya no se trata de las circunstancias del afuera, sino de algo que forma parte y es inherente al propio aparato psíquico, como constitutivo de su ser.

La idea de locura está vinculada a la producción de un estado caótico, descontrolado, confusional, en el cual no hay patrones que puedan implementarse para frenar la pérdida de referentes de la realidad. En la fantasía, se podría estar en condiciones de poder realizar casi cualquier cosa, sin control y sin voluntad. La presencia misma del impulso que se impone.

La locura está asociada al desenfreno y descontrol de la sexualidad. El placer sería tan intenso que rompería todo muro de contención. Sería el ejercicio del puro placer que operaría sobre la anulación de todo pensar. La locura aparece como un momento de desintegración, de estallido de la personalidad lo que produciría intensísimas angustias ante la fantasía de un viaje sin retorno. Ante dicha ruptura yoica, dominarían ansiedades de persecusión y ataque hasta el exterminio.

Las defensas, por tanto, deben desde un inicio "enfrentar" al paciente para lograr su control, para evitar la contaminación del espacio de la entrevista que dejaría secuelas imprevisibles en la figura del estudiante. Solamente la permeabilidad yoica ante los miedos personales del practicante, puede producir un acercamiento menos persecutorio a la figura del paciente. De lo contrario, el "encuentro" con el paciente se constituirá en una experiencia traumática.

En todo caso, este es el factor determinante que desencadenaría la necesidad de psicoterapia personal de todo futuro clínico. La experiencia muestra allí que la figura del supervisor es esencial para objetivar ciertas confusiones entre la patología del "paciente" y la del "entrevistador", sugiriendo la posibilidad de un trabajo psicoterapéutico personal.

\section{Evaluación grupal}

Finalizadas las sesiones tiene lugar una última reunión de evaluación grupal. Se trata de que el grupo construya su propia autoevaluación, reflexionando sobre lo acontecido y acerca de lo que la experiencia grupal le dejó a cada quien. A través de sus comentarios van reconociendo:

- que el proceso grupal desarrollado guarda semejanzas con el proceso de la cura y que el darse cuenta del propio lugar en el grupo les ayuda a poder pensarse en la relación con el paciente: "la paciencia necesaria".

- que el espacio grupal abre a una horizontalidad pensante que legitima el aprendizaje colectivo, poder escuchar a otros es también escucharse a sí mismo. Relacionarse con "otro que piensa distinto de ti" inicia también la función de supervisión y la posibilidad de auto-observación, "la angustia es siempre de uno".

- que el espacio de reflexión posibilitó ir analizando algunos miedos que todos tenían, incluso al punto de producirse un cambio radical ("me bajó caleta la ansiedad"): alguien que al inicio no se animaba a aceptar un paciente pudo no solamente enfrentarse a la situación sino que también pensar algo durante la entrevista.

- que se desarrolló de manera muy importante la "agudeza de observación”. Los señalamientos e interpretaciones realizados durante las sesiones objetivan fenómenos que pueden ser internalizados por los alumnos como una manera de aprehender el objeto y el proceso de trabajo. Este aspecto se ve apoyado por el desarrollo de la capacidad para tomar distancia del objeto manteniendo una reacción afectiva que no los inhabilita para pensar. "Uno no se puede escapar del paciente", señalan. Otro integrante habló de la "imposición de intolerancia" cuando deben escuchar expresiones y frases con las que discrepan.

- que en base al aprendizaje grupal realizado les es posible "tolerar el silencio" del paciente y "no abrumarlo con intervenciones y preguntas".

- que hay una carencia de espacios apropiados para hablar del paciente y de todo lo que "se le produce a uno en la entrevista". Es reconocer la necesidad de un espacio de contención y reflexión, propuesta de simbolización para que la experiencia clínica que se inicia aporte a la salud mental del estudiante.

\section{Conclusiones}

El grupo de reflexión conducido con la técnica operativa de grupo ha demostrado ser un medio apropiado para 
facilitar la incorporación de estudiantes de psicología a la práctica de la psicología clínica: realización de primeras entrevistas y sesiones de psicoterapia, sobre todo para evitar que una experiencia tan significativamente afectiva como la relatada se pueda inscribir en su psiquismo como traumática.

La posibilidad que tienen los estudiantes que realizan sus primeros "enfrentamientos" con pacientes de verse afectados por un encuentro emocional que los desborde es bastante alta si se toma en cuenta, por ejemplo, el estudio realizado por Cova, F. y colabs (2007) con estudiantes universitarios, que muestra un alto índice de sintomatología ansiógena.

A su vez, como lo muestra la evaluación grupal, el espacio grupal de reflexión les permite un espacio que tiene dos perfiles:

1. Funciona con todas las características de un espacio clínico propio sin fijarse un objetivo terapéutico. Esto autoriza la puesta en juego del mecanismo de disciminación e identificación que contribuye a una mejor ubicación en el rol de psicólogo.

2. Provee de una experiencia, amplia y global, rica en estímulos y en detalles, que agudiza la observación y facilita la toma de distancia adecuada para una inclusión personal en la misma resultando en un aprendizaje de la clínica como práctica.

\section{Referencias}

Bleger, J. (1961). Grupos operativos en la enseñanza. Temas de psicología. Buenos Aires: Nueva visión

Cabeza, V. \& Foladori, H. (1978). Grupos psicoanalíticos terapéuticodidácticos en la enseñanza universitaria, Enseñanza e Investigación en Psicología, 4, 25-33.

Cova, F. (2007). Problemas de salud mental en estudiantes de la Universidad de Concepción, Terapia Psicológica, 25, 105-112.

Etchegoyen, H. (1959) Psicoterapia de grupo en la enseñanza médica. En: Grinberg, L., Langer,M. \& Rodrigué, E., El grupo psicológico. 234-246. Buenos Aires: Ed. Nova
Dellarossa, A. (1979) Grupos de reflexión, Bs.As.: Paidós

Foladori, H. (1984). Una experiencia didáctica grupal: entrenamiento en entrevista abierta. El grupo operativo de-formación. (133-143). Santiago: U. Bolivariana

Foladori, H. (1999) Dispositivos, encuadres operativos y otras yerbas. $E l$ grupo operativo de-formación (106-120). Santiago: U. Bolivariana.

Foladori, H. (2000) Psicoanálisis grupal para estudiantes de psicología. Memorias de las Jornadas Clínicas del Caps. (13-20) Santiago: Universidad de Chile.

Foladori, H. (2001) El grupo operativo en el marco de la educación superior. El grupo operativo de-formación. (38-54) Santiago: U. Bolivariana

Ganzarain, R. \& Davanzo, H. (1957). Evolución después de dos años, de los resultados obtenidos en psicoterapia de grupo en la formación psiquiátrica en estudiantes de medicina. Evaluación del aprendizaje sobre transferencia obtenido mediante la psicoterapia colectiva. Primer congreso latinoamericano de psicoterapia de grupo. Buenos Aires: UBA

Ganzarain, R., Davanzo, H. \& Cizaletti, J. (1958) Group Psychotherapy in the Psychiatric Training of Medical Studentes. The International Journal of Group Psychotherapy, 8(2), 137-153.

Ganzarain, R.(1959). Aplicaciones de la psicoterapia de grupo en la enseñanza de la psiquiatría. En Grinberg, L., Langer, M. \& Rodrigué, E. El grupo psicológico (247-288) Buenos Aires: Ed. Nova

Ganzarain, R., Davanzo, H., Flores, O. \& Drobny, E. (1959). Study of Effectiveness of Group Psychotherapy in the Training of Medical Studentes, The International Journal of Group Psychotherapy, 9 (4), 475-487.

Grinberg, L., Langer, M. \& Rodrigué, E. (1957). Psicoterapia del grupo. Buenos Aires: Paidós

Langer, M. (1948) Dificultades psicológicas del psicoanalista principiante. Revista de Psicoanálisis, 20, 333-345.

Langer, M. (1977). Experiencias con grupos terapéutico-didácticos con estudiantes de psicología en el marco de su formación universitaria. Imago, 2, 37-44.

Pichón-Rivière, E. \& Etchegoyen, H. (1957) La psiquiatría en el contexto de los estudios médicos. El proceso grupal. Bs.As.: Nueva visión, 1983

Pichón-Rivière, E. (1971). Del psicoanálisis a la psicología social. Buenos Aires: Galerna

Saretta, J. \& Veloz, R.M. (1989) Importancia del grupo de reflexión para la formación del psicoterapeuta psicoanalítico. Revista de Psicoterapia, Psicoanálisis y Psiquiatría, 10, 123-139.

Ulloa, F. (1973). Grupo de reflexión y ámbito institucional en los programas de promoción y prevención de la salud. En: Grimson, E. Nuevas perspectivas en salud mental. Institucionales y problemas (119-139). Buenos Aires: Nueva visión. 